ThE Astrophysical JouRNAL, 525:734-736, 1999 November 10

(c) 1999. The American Astronomical Society. All rights reserved. Printed in U.S.A.

\title{
A NEW ARGUMENT AGAINST AN INTERVENING STELLAR POPULATION TOWARD THE LARGE MAGELLANIC CLOUD
}

\author{
ANDREW GOULD \\ Department of Astronomy, Ohio State University, 174 West 18th Avenue, Columbus, OH 43210; gould@astronomy.ohio-state.edu \\ Received 1999 February 26; accepted 1999 June 25
}

\begin{abstract}
Zaritsky \& Lin have claimed detection of an intervening population of stars toward the Large Magellanic Cloud (LMC), which, they believe, could account for a substantial fraction of the observed microlensing events. I show that the observed timescales of these events imply that if such an intervening population were composed of ordinary stars that gave rise to a significant fraction of the microlensing events, then the population could not be associated with the LMC. I present two independent statistical arguments which together essentially rule out such a chance alignment of unassociated structures. On the other hand, if the intervening structure is associated with the LMC, I show that of order half the mass in this structure is in substellar objects, which would make it unlike any known stellar population.

Subject heading: dark matter — Galaxy: halo — gravitational lensing — Magellanic Clouds
\end{abstract}

\section{INTRODUCTION}

Zaritsky \& Lin (1997) found a concentration of stars approximately 0.9 mag above the red clump (RC) in a colormagnitude diagram (CMD) of the Large Magellanic Cloud (LMC). They suggested that this concentration of stars might trace a foreground population of ordinary stars, and this foreground population might be responsible for a large fraction of the microlensing events seen toward the LMC by Alcock et al. (1997a) and Aubourg et al. (1993).

A number of workers raised a diverse set of objections to this hypothesis. Alcock et al. (1997b) showed that if the putative foreground population lay within $33 \mathrm{kpc}$ (i.e., 0.9 mag for an assumed LMC distance of $50 \mathrm{kpc}$ ), then it contained no detectable population of RR Lyrae stars. Beaulieu \& Sackett (1998) showed that a vertical red clump (VRC), that is, a vertical extension to the usual red clump, is a typical feature of CMDs for populations of mixed age, and hence the presence of a VRC did not necessarily indicate a foreground population. Gallart (1998) showed that such features are present in the Fornax and Sextans A dwarf galaxies. I argued (Gould 1998) that if such a foreground structure were composed of tidal debris, then either it should have shown up in de Vaucouleurs's (1957) map of the LMC, or it must have an anomalously high mass-tolight $(M / L)$ ratio to account for the microlensing events. Johnston (1998) showed that tidal debris from disrupted satellites would give rise to unacceptably high star counts away from the LMC if it were to account for the microlensing events seen toward the LMC. Bennett (1998) related the surface density of RC stars to the total surface mass density of their parent stellar distribution and showed that for typical stellar populations the density of the VRC reported by Zaritsky \& Lin (1997) was too low by an order of magnitude to account for the microlensing.

Zaritsky et al. (1999) have addressed each of the objections in turn. They said that it was possible to construct an initial mass function with a much higher ratio of total mass to RC stars than for the "typical " parameters advocated by Bennett (1998). They argued that the foreground population could be at $40 \mathrm{kpc}$, rather than the $33 \mathrm{kpc}$ originally proposed by Zaritsky \& Lin (1997), thus evading the constraint of Alcock et al. (1997b). They pointed out that while certain star formation histories could well explain the VRC as a feature of the LMC CMD as advocated by Beaulieu \& Sackett (1998), such histories were not demanded by the available data, and indeed an independently constructed history yields only a small fraction of the observed VRC. They argued that Johnston's (1998) analysis does not apply to tidal material from an SMC-LMC interaction or from a denser than expected LMC halo. Finally they quoted from de Vaucouleurs (1957) to make it appear that he himself did not believe the outer isophotes of his map, thereby apparently dispensing with my argument (Gould 1998).

It is not my purpose here to examine critically all of these counterarguments, a task which would require a major investigation in its own right. Rather, I present a new argument against the hypothesis that the VRC traces a significant lensing population.

\section{TRANSVERSE SPEED OF THE LENSES}

The speed of the lenses relative to the observer-source line of sight, $v_{\perp}$, is related to the observed timescale of the events $t_{\mathrm{E}}$ by

$$
\begin{aligned}
v_{\perp} & =190 \mathrm{~km} \mathrm{~s}^{-1}\left(\frac{t_{\mathrm{E}}}{40 \text { days }}\right)^{-1}\left(\frac{M}{0.25 M_{\odot}}\right)^{1 / 2}\left(\frac{\hat{D}}{10 \mathrm{kpc}}\right)^{1 / 2} \\
\hat{D} & \equiv \frac{d_{\mathrm{ol}} d_{\mathrm{ls}}}{d_{\mathrm{os}}}
\end{aligned}
$$

where $M$ is the mass of the lens, and $d_{\mathrm{ol}}, d_{\mathrm{ls}}$, and $d_{\mathrm{os}}$ are the respective distances between the observer, lens, and source. This equation summarizes the major difficulty in explaining the lenses as halo objects: if the lenses were in the halo $(\hat{D} \sim 10 \mathrm{kpc})$ and were substellar objects $\left(M<0.08 M_{\odot}\right)$, then to produce events with the observed timescales $\left(t_{\mathrm{E}} \sim\right.$ 40 days), they must be moving with typical speeds $v_{\perp} \lesssim 110$ $\mathrm{km} \mathrm{s}^{-1}$, which is more than a factor of 2 smaller than the speeds expected from halo dynamics. Hence, if they are in the halo, they are not made of hydrogen: substellar objects would be moving too slowly, while stellar objects made of hydrogen would burn and be visible (e.g., Gould, Flynn, \& Bahcall 1998 and references therein).

A somewhat technical but important point is that a characteristic timescale estimate of $t_{\mathrm{E}} \sim 40$ days in equation (1) comes from the average of the timescales of the eight events 
observed by Alcock et al. (1997a). The distribution of these events is of course affected by selection, i.e., by the efficiency of detecting events as a function of their timescale. This efficiency peaks at $\sim 40$ days, which is suspiciously close to the average observed value. However, the efficiency is quite broad: it is above half its peak value on the interval 6 days $\lesssim t_{\mathrm{E}} \lesssim 125$ days, whereas the observed events cover only the range 17 days $\leq t_{\mathrm{E}} \leq 72$ days. Therefore, the estimate of the typical timescale is not likely to be highly biased by selection unless there is an entirely different population of lenses whose timescales are disjoint from the observed ones.

Equation (1) can also be used to draw significant conclusions about the putative foreground structure claimed by Zaritsky \& Lin (1997). If this structure is composed of ordinary stars $\left(M \sim 0.25 M_{\odot}\right)$, and if it lies $0.9 \mathrm{mag}(17 \mathrm{kpc})$ in front of the $\operatorname{LMC}(\hat{D}=11 \mathrm{kpc})$, then it must be traveling at $v_{\perp} \sim 200 \mathrm{~km} \mathrm{~s}^{-1}$ relative to the line of sight to the LMC. This is approximately what would be expected for a random object traveling through the Galactic halo, such as a dwarf galaxy or tidal debris from a disrupted dwarf (Zhao 1998). However, it is substantially too high for material associated with the LMC.

Hence, if the claimed foreground structure is truly responsible for a substantial fraction of the microlensing events, then there are two possibilities: either the foreground structure is not associated with the LMC, or it is associated but is composed of objects that are substantially lighter than the mass of typical stars, $M \sim 0.25 M_{\odot}$. I examine these two possibilities in turn, beginning with the hypothesis of a chance alignment of a structure unassociated with the LMC.

The a priori probability of such an alignment is incredibly small. Recall from Gould (1998) that the surface mass density required to explain the observed microlensing optical depth, $\tau \sim 2.9 \times 10^{-7}$, is

$$
\Sigma=47\left(\frac{\tau}{2.9 \times 10^{-7}}\right)\left(\frac{\hat{D}}{10 \mathrm{kpc}}\right)^{-1} M_{\odot} \mathrm{pc}^{-2} .
$$

The optical depth estimate from Alcock et al. (1997a) is much larger than the optical depths expected from selflensing by the LMC disk ( $\tau \sim 2 \times 10^{-8}$; Gould 1995), from lensing by the Milky Way disk $\left(\tau \sim 8 \times 10^{-9}\right.$; Gould, Bahcall, \& Flynn 1997), and by the Milky Way spheroid ( $\tau \sim 5 \times 10^{-9}$; Gould, Flynn, \& Bahcall 1998). I therefore ignore all these minor adjustments to equation (2). On the other hand, I should caution that the estimate itself is based on only eight events, and therefore is subject to a very large statistical error, specifically $\tau \sim 2.9_{-0.9}^{+1.4} \times 10^{-9}$ (Alcock et al. 1997a).

For a Milky Way disk with $M / L=1.8$, the surface density in equation (2) corresponds to about $V=22.8 \mathrm{mag}$ $\operatorname{arcsec}^{-2}$. By comparison, the central surface brightnesses of the Sculptor and Sextans dwarf spheroidal galaxies are respectively 23.7 and $26.1 \mathrm{mag} \operatorname{arcsec}^{-2}$ (Mateo et al. 1991). Moreover, the core radii of these galaxies are only $9^{\prime}$ and $15^{\prime}$, respectively (Mateo et al. 1991), much smaller than the several square degrees required to account for the microlensing events. The fraction of the high-latitude sky covered by dwarf galaxies of even these low surface brightnesses is well under $10^{-4}$. (Note that the a priori probability of a structure associated with the $L M C$ would not be affected by this argument.)

\section{EXPECTED RADIAL VELOCITY DIFFERENCE}

It is not, however, entirely appropriate to apply a priori statistical arguments to the presence of a dwarf galaxy in front of the LMC. The fact is that microlensing events have been discovered toward the LMC, and all the explanations offered so far are a priori unlikely. If evidence is produced for an intervening stellar population after the detection of the microlensing events (e.g., Zaritsky \& Lin 1997), then the low a priori probability for such a population carries less weight.

Nevertheless, this putative detection brings with it the means for an additional, truly a priori test. If the intervening population is not associated with the LMC, then its radial motion relative to the LMC should be a random value drawn from a distribution characteristic of Galactic satellites. On the other hand, if the VRC is actually composed of LMC stars, the two radial velocities should be consistent.

I find that the rms Galactocentric radial velocity of 16 satellite galaxies and distant globular clusters at high latitude (422-213, AM 1, Carina, Draco, Fornax, LMC, Leo I, Leo II, NGC 2419, Pal 3, Pal 4, Pal 14, Sculptor, Sextans, $\mathrm{SMC}$, and UMi) is $\sigma_{\text {sat }}=86 \mathrm{~km} \mathrm{~s}^{-1}$. Zaritsky et al. (1999) have measured the mean radial velocities of the two populations and find a difference,

$$
\Delta \bar{v}=\bar{v}_{\mathrm{VRC}}-\bar{v}_{\mathrm{RC}}=5.8 \pm 4.9 \mathrm{~km} \mathrm{~s}^{-1},
$$

where (in contrast to Zaritsky et al. 1999) I am quoting $1 \sigma$ errors. This confirms the results of Ibata, Lewis, \& Beaulieu (1998) based on a smaller sample and is just what one would expect if the VRC stars were part of the LMC. However, the a priori probability that the two populations would be this close if they were not associated is

$$
p \sim \frac{2 \Delta \bar{v}}{(2 \pi)^{1 / 2} \sigma_{\mathrm{sat}}} \exp \left(-\frac{v_{\mathrm{LMC}}^{2}}{2 \sigma_{\mathrm{sat}}^{2}}\right) \sim 4 \%,
$$

where $v_{\mathrm{LMC}}=73 \mathrm{~km} \mathrm{~s}^{-1}$ is the Galactocentric radial velocity of the LMC.

In brief, there are two distinct statistical arguments against the VRC being a foreground structure that is not associated with the LMC: first, it is unlikely $\left(<10^{-4}\right)$ that such a structure would happen to be aligned with the LMC; second, it is unlikely (4\%) that it would have a radial velocity consistent with that of the LMC. Together, these two arguments effectively rule out this possibility.

\section{MASS SCALE OF FOREGROUND POPULATION}

I therefore turn to the second possibility discussed in $\S 2$, that the masses of the foreground objects are substantially smaller than those of typical stars. To investigate this possibility, it is first necessary to estimate the typical transverse velocities of populations that are associated with the LMC. For a foreground population with the same space velocity as the LMC but lying a distance $d_{\mathrm{ls}}$ in front of it, the transverse speed of the lens population relative to the EarthLMC line of sight is

$$
v_{\perp, \text { bulk }}=\mu_{\mathrm{LMC}} d_{\mathrm{ls}}=109 \mathrm{~km} \mathrm{~s}^{-1} \frac{d_{1 \mathrm{~s}}}{17 \mathrm{kpc}},
$$

where $\mu_{\mathrm{LMC}}=1.35$ mas $\mathrm{yr}^{-1}$ is the proper motion of the LMC (Jones, Klemola, \& Lin 1994). To find $v_{\perp}$, this bulk 
motion must be added to any peculiar motion of the sources and lenses relative to the assumed common space motion. The internal dispersion of the VRC is small $\left(18 \mathrm{~km} \mathrm{~s}^{-1}\right.$; Zaritsky et al. 1999) and therefore can be ignored. The bulk motion of the foreground structure relative to the LMC must also be small to evade the arguments given in the last two sections about unassociated structures, so this will also be ignored. However, the LMC sources are rotating at $\sim 70$ $\mathrm{km} \mathrm{s}^{-1}$, and this must be included. ${ }^{1}$ It should first be multiplied by the projection factor $d_{\mathrm{ol}} / d_{\mathrm{os}}$ and then added in quadrature (because the microlensing observations cover a sufficiently large part of the LMC that all directions of motion are effectively covered). Hence, at $d_{\mathrm{ls}}=17 \mathrm{kpc}$, the expected $v_{\perp}$ is $119 \mathrm{~km} \mathrm{~s}^{-1}$. Inserting this value in equation (1) yields a typical mass $M \sim 0.09 M_{\odot}$ at $d_{1 \mathrm{~s}}=17 \mathrm{kpc}$. At $d_{1 \mathrm{~s}}=10 \mathrm{kpc}$, the same argument yields $M \sim 0.06 M_{\odot}$.

${ }^{1}$ The estimate of $v_{\text {rot }} \sim 70 \mathrm{~km} \mathrm{~s}^{-1}$, while conventional, is somewhat uncertain. The projected rotation $v_{\text {rot }} \sin i$ (where $i$ is the inclination) has been measured for various populations, including $28 \mathrm{~km} \mathrm{~s}^{-1}$ for $\mathrm{H}$ I and 39 $\mathrm{km} \mathrm{s}^{-1}$ for CO. See Hughes, Wood, \& Reid (1991) for a summary. If these are deprojected using the conventional $i=27^{\circ}$, one derives $v_{\text {rot }} \sim 60$ and $80 \mathrm{~km} \mathrm{~s}^{-1}$, respectively. However, a larger inclination, e.g., $45^{\circ}$, which is sometimes discussed, would yield values that were lower by a factor of 0.65 . If $v_{\text {rot }}$ were lower than assumed here, the inferred mass scale of the lenses would be even lower than the estimate I derive below.
Another possibility for a foreground population at $d_{1 \mathrm{~s}}=10$ $\mathrm{kpc}$ is that it is a bound satellite orbiting about the LMC at $\sim 70 \mathrm{~km} \mathrm{~s}^{-1}$. However, this scenario leads to essentially the same mass, $M \sim 0.07 M_{\odot}$. For distances $d_{1 \mathrm{~s}} \ll 10 \mathrm{kpc}$, it is no longer plausible that the foreground population would give rise to the observed VRC, which peaks 0.9 mag brighter than the RC. Thus, $M \sim 0.08 M_{\odot}$ is a robust estimate of the characteristic mass of the putative foreground population.

Since $M \sim 0.08 M_{\odot}$ is approximately where hydrogen burning begins, this result implies that of order half the mass in the putative foreground structure lies below the hydrogen-burning limit. While this is possible in principle, it should be noted that in the solar neighborhood, substellar objects account for only about one-sixth of the total stellar and substellar mass (Holmberg \& Flynn 1999 and references therein).

I thank B. S. Gaudi for a careful reading of the manuscript, and D. Zaritsky for critical but very valuable comments. This work was supported in part by grant AST 97-27520 from the NSF and in part by grant NAG5-3111 from NASA.
Alcock, C., et al. 1997a, ApJ, 486, 697
Aubourg., E., et al. 1993, Nature, 365, 623
Beaulieu, J.-P., \& Sackett, P. D. 1998, AJ, 116, 209
Bennett, D. 1998, ApJ, 493, L79
de Vaucouleurs, G. 1957, AJ, 62, 69
Gallart, C. 1998, ApJ, 495, L43
Gould, A. 1995, ApJ, 441,777
Gould, 1998, ApJ, 499, 728
Goul, Bahcall, J. N., \& Flynn, C. 1997, ApJ, 482, 913
Gould, A., Flynn, C., \& Bahcall, J. N. 1998, ApJ, 503, 798

\section{REFERENCES}

Holmberg, J., \& Flynn, C. 1999, MNRAS, submitted Hughes, S. M. G., Wood, P. R., \& Reid, N. 1991, AJ, 101, 1304 Ibata, R. A., Lewis, G. F., \& Beaulieu, J.-P. 1998, ApJ, 509, L29 Johnston, K. V. 1998, ApJ, 495, 297

Jones, B. F., Klemola, A. R., \& Lin, D. N. C. 1994, AJ, 107, 1333

Mateo, M., Nemec, J., Irwin, M., \& McMahon, R. 1991, AJ, 101, 892 Zaritsky, D., \& Lin, D. N. C. 1997, AJ, 114, 2545

Zaritsky, D., Shectman, S. A., Thompson, I., Harris, J., \& Lin, D. N. C. 1999, AJ, 117, 2268

Zhao, H. 1998, MNRAS, 294, 139 